\title{
El Aprendizaje Cooperativo en la clase de Educación Física: dificultades iniciales y propuestas para su desarrollo
}

Martínez Benito, Raúl; Sánchez Sánchez, Galo

El Aprendizaje Cooperativo en la clase de Educación Física: dificultades iniciales y propuestas para su desarrollo Revista Educación, vol. 44, núm. 1, 2020

Universidad de Costa Rica, Costa Rica

Disponible en: http://www.redalyc.org/articulo.oa?id=44060092003

DOI: https://doi.org/10.15517/revedu.v44i1.35617

Esta obra está bajo una Licencia Creative Commons Atribución-NoComercial-SinDerivar 3.0 Internacional. 


\section{El Aprendizaje Cooperativo en la clase de Educación Física: dificultades iniciales y propuestas para su desarrollo}

Cooperative Learning in Physical Education: Initial Challenges and Developmental Proposals

Raúl Martinez Benito

Universidad de Salamanca, España

raul.martinez@usal.es

(iD http://orcid.org/0000-0002-4989-4426

Galo Sánchez Sánchez

Universidad de Salamanca, España

galo@usal.es

(iD http://orcid.org/0000-0003-3048-247X
DOI: https://doi.org/10.15517/revedu.v44i1.35617

Redalyc: http://www.redalyc.org/articulo.oa?id=44060092003
Recepción: 14 Enero 2019

Aprobación: 07 Julio 2019

\section{Resumen:}

El presente artículo forma parte de una tesis en elaboración en la cual se investiga los factores de eficacia del Aprendizaje Cooperativo (AC) en Educación Física (EF). El objetivo es mostrar los resultados de una primera revisión, sobre los problemas que encuentra el profesorado al aplicar esta metodología; y unido a ello, presentar las principales propuestas encontradas para su aplicación en la clase de EF de una manera progresiva y eficaz. Se ha procedido al vaciado de fuentes documentales, para posteriormente catalogar y clasificar aquellos aspectos objetos del estudio: problemas y propuestas de implementación. Se acudió a las siguientes bases de datos: Teseo, Google Académico, Dialnet, EBSCOhost, Scopus y Web of Science. La revisión fue desarrollada a finales del año 2018. Los resultados demuestran que varias dificultades pueden aparecer al aplicar esta metodología. Se han agrupado en siete categorías: 1) Falta de experiencias y habilidades del alumnado; 2) Experiencias negativas previas del alumnado;3) Falta de formación del profesorado; 4) Problemas de organización a nivel de centro; 5) Oposición familiar; 6) Falta de control de aula; y 7) Expectativas poco realistas del cuerpo docente. En lo relativo a las propuestas de aplicación, se han encontrado dos que presentan un proceso concreto para llevar a cabo la metodología de una manera progresiva y eficaz en EF, definiendo las variables a influir y las condiciones necesarias a generar, para minimizar o solucionar los problemas iniciales: El enfoque de la Coopedagogía Motriz (Velázquez, 2014, 2015, 2018), y el Ciclo del Aprendizaje Cooperativo (Iglesias, González y Fernández, 2017).

Palabras Clave: Educación Física, Aprendizaje Cooperativo, Modelos pedagógicos, Implementación Aprendizaje Cooperativo.

\section{Abstract:}

This article is part of a thesis that studies efficiency factors of Cooperative Learning in Physical Education. The objective is to present the results of a review about constraints faced by teachers who use this strategy and, in addition, show how it can be used progressively and effectively in PE classes. A bibliographic review was conducted revealing challenges faced by teachers who use Cooperative Learning strategies. The review included searches in the following databases in late 2018: Teseo, Google Scholar, Dialnet, EBSCOhost, Scopus and Web of Science. Results show that when using this strategy, certain difficulties may arise which we classified into seven categories: 1) Lack of student skills and experience; 2) Previous negative experience of the student; 3) Lack of training among the teachers; 4) Organizational problems at the school; 5) Family opposition; 6) Teacher cannot control classroom behavior; and 7) Unrealistic teacher expectations. Two proposals for executing the Cooperative Learning in PE were found to be progressive and effective. They set variables that influence cooperative learning as well as the necessary conditions to minimize or resolve problems at the onset: The Motor Coopedagogy Approach (Velázquez, 2014, 2015, 2018), and the Cooperative Learning Cycle (Iglesias, González and Fernández, 2017).

KEYWORDS: Physical Education, Cooperative Learning, Pedagogical Models, Cooperative Learning Implementation. 


\section{INTRODUCCIÓN}

En las guerras unos dan discursos y otros se juegan la vida en el frente de batalla. Esta frase, extraída de la obra de teatro Viriato, de Florián Recio (VerboProducciones, 2017) resulta perfectamente extrapolable al ámbito de la educación. A veces, los maestros y las maestras se sienten así, cuando acuden a cursos, charlas, seminarios, congresos o simplemente escuchan, o leen a ponentes que teorizan sobre el qué hacer y cómo hacer en el aula. En ocasiones esta teoría no resulta suficiente para aplicarla de manera efectiva en sus clases, pues está basada en contextos ideales o muy diferentes a los que después realmente se encuentran, se centran únicamente en los aspectos positivos, obviando los problemas que aparecerán por el camino, o los pasos necesarios para su implementación progresiva y segura. Además, no estamos hablando de un modelo sencillo, pues el Aprendizaje Cooperativo (AC) utiliza grupos de trabajo pequeños, generalmente heterogéneos, donde todos los miembros comparten esfuerzos para aumentar el conocimiento propio y el de los compañeros (Dyson, 2002; Johnson, Johnson y Holubec, 1999; Velázquez, 2013a, 2013b). En otras palabras, el estudiantado tiene una doble responsabilidad, teniendo que preocuparse también del aprendizaje de sus compañeros, siendo este un aspecto que aumenta su complejidad.

Velázquez (2013a) establece que el AC, a la vista de las numerosas experiencias e investigaciones publicadas, es un modelo pedagógico en auge en los últimos años. Sin embargo, a pesar de la cantidad de información que poseemos, su puesta en práctica no resulta una tarea tan sencilla. En este sentido, son muchos los autores que han investigado, experimentado o reflexionado sobre los principales problemas que se pueden encontrar al plantear esta metodología (Fernández, 2003; Iglesias et ál., 2017; Johnson, Johnson y Holubec, 1999; Marín y Blázquez, 2003; Martín, 2014; Poveda, 2006; Prieto, 2007; Velázquez, 2004, 2009, 2010, 2013a), que se caracteriza porque los estudiantes interaccionan entre sí con el objetivo de adquirir aprendizajes, pero con la responsabilidad añadida de que sus compañeros también aprendan.

Este trabajo tiene como objetivo describir los principales problemas que pueden encontrarse al aplicar el AC, además de presentar las principales propuestas encontradas para su aplicación en la clase de Educación Física (EF) de una manera progresiva y eficaz.

\section{CONTEXTUALIZACIÓN Y FINALIDADES DEL ARTÍCULO}

El siguiente artículo forma parte de una tesis en elaboración que trata de investigar sobre los factores de eficacia en la aplicación del AC en EF. En el artículo se expondrán los resultados de una primera revisión bibliográfica. A través de esta exploración inicial se pretende tener una primera idea de los posibles problemas que puede encontrarse el profesorado de EF que utiliza el AC. Por otro lado, y estrechamente vinculado con el anterior, se ha tratado de encontrar propuestas que, con un carácter concreto y progresivo, establezcan una serie de fases para aplicar esta metodología en el aula de una manera progresiva, evitando o minimizando de esta manera los problemas que pueden ir apareciendo en el proceso.

En un primer momento, se ha efectuado una búsqueda de tesis doctorales (publicadas en España), pertenecientes al AC en EF. Para ello, se han cruzado los términos aprendizaje cooperativo y educación física en una búsqueda efectuada a través de las bases de datos Teseo, Dialnet y Google Académico. El resultado nos ha indicado que dos trabajos cumplían los criterios establecidos: las tesis doctorales de Fernández (2003) y Velázquez (2013a).

La revisión de ambas, junto con sus referencias bibliográficas, han servido como guía a la hora de seleccionar lecturas posteriores. Aunque, en un primer momento se pretendía únicamente revisar documentos relacionados con el área de $\mathrm{EF}$, finalmente, debido a la escasez en los que se hacía referencia a los aspectos objetos del estudio (problemas y propuestas de desarrollo), también se han revisado otros que tratan el AC de una manera más general, o que tienen relación con otras áreas. 
Esta primera fase de la revisión se ha complementado con la lectura posterior de documentos que hace referencia al AC en EF. Para ello, se han vuelto a cruzar en otra búsqueda los términos educación física y aprendizaje cooperativo, acotándola a publicaciones a partir del año 2013. Con este filtro temporal se pretende revisar aquellos documentos publicados a partir de la última tesis doctoral revisada. Esta segunda búsqueda se ha llevado a cabo en las bases de datos Google Académico, Dialnet, EBSCOhost, Scopus y Web of Science.

\section{Resultados}

A continuación, se muestran los resultados de esta primera revisión bibliográfica. Se han dividido en dos apartados, correspondiendo cada uno de ellos a los objetos de la investigación:

\section{Problemas al aplicar el aprendizaje cooperativo}

Colocar al alumnado en grupos no es suficiente para conseguir su aprendizaje y desarrollo personal. No es la propia interacción la que produce resultados beneficiosos, sino la naturaleza de esta interacción (Iglesias et ál., 2017). Por lo tanto, es un error creer que disponerles en pequeños grupos y proponerles una tarea debe generar necesariamente que cooperen con eficacia (Velázquez, 2010). En este sentido, a continuación se describen algunos de los problemas más habituales que suelen aparecer cuando se agrupan y tienen que interaccionar con compañeros:

1. Falta de experiencia y de habilidades del alumnado:

Compartir el aprendizaje y trabajo con otros no es una tarea sencilla, pues no se trata de una característica innata en el ser humano, y no es algo que se aprenda por intuición o de manera espontánea. No se accede a la escuela sabiendo interactuar correctamente con las demás personas, siendo necesario enseñar a los estudiantes una serie de destrezas y actitudes necesarias para la cooperación. (Iglesias et ál., 2017; Johnson et ál., 1999; León, 2002; Velázquez, 2013a).

Estudiantes poco habituados a cooperar pueden mostrar tendencia a comportarse de manera individualista o competitiva, perjudicando de esta manera las respuestas cooperativas de otros, rechazando a personas que consideran incompetentes para ejecutar la tarea asignada, descentrándose cuando trabajan en grupo o comparando los resultados entre personas o grupos (Velázquez, 2018). Un estudio de caso múltiple elaborado con siete expertos en AC concluyó que este tipo de mentalidades manifestadas en el aula por el estudiantado era uno de los principales problemas que se encontraba el profesorado (Velázquez, 2013a).

Tampoco ayuda el contexto social en el cual vivimos, donde los valores predominantes se asientan en la competitividad y el individualismo (Iglesias et ál., 2017; Marín y Blázquez, 2003; Velázquez, 2009). En muchas ocasiones, este contexto también se reproduce en la escuela, por lo que resulta complicado esperar del alumnado una forma de trabajar a la que no está acostumbrado (Prieto, 2007; Velázquez, 2018).

2. Experiencias negativas previas:

Como señala Ovejero (1990), se necesita que tanto docentes como estudiantes quieran trabajar cooperativamente, y esto no siempre ocurre. En ocasiones, aquellos alumnos o alumnas que en formas de trabajo tradicional tienen éxito, pueden sentirse menos seguros y más incómodos en estas situaciones de trabajo en grupo. Otras veces, algunos manifiestan resistencia a trabajar en grupos cooperativos, porque se han cansado de ayudar a aquellos que no quieren ser ayudados, o que tienen poco interés por aprender (Marín y Blázquez, 2003). Incluso puede ocurrir que tengan "viejos problemas de relación interpersonal, existan rivalidades y desconfianzas o acumulen experiencias personales negativas de exclusión” (Iglesias et ál., 2017, p. 62). 
3. Falta de formación del profesorado:

En 1998 Lobato (citado por Prieto, 2007, p.35), señala la falta de formación del profesorado como un inconveniente a la hora de llevar a cabo este tipo de metodologías. Además de la falta de recursos bibliográficos, analizando los programas de estudio para profesores de Primaria y Secundaria, se puede observar una falta de formación inicial con respecto a metodologías cooperativas (Velázquez, 2010, 2013a, 2018).

4. Problemas de organización a nivel de centro y aula:

El propio currículum, el horario establecido en el centro o la organización del aula, pueden añadir obstáculos a la hora de aplicar el AC. La distribución de las mesas, el excesivo ruido, o la falta de espacio para que cada grupo pueda tener un mínimo de privacidad para el desarrollo de la tarea pueden constituir condicionantes de tipo organizativo (Marín y Blázquez, 2003).

5. Oposición familiar:

Puede ocurrir que sean las propias familias las que rechazan esta manera de trabajar, en unas ocasiones debido al peso de la actual cultura competitiva e individualista en la que vivimos, y en otras, aludiendo a su propia experiencia como alumnos (Marín y Blázquez, 2003).

Ciertamente, esta oposición subyace en una serie de falsas creencias que relacionan la competición con el éxito, con la diversión, o con la construcción del carácter. En este sentido, se defiende una escuela donde se formen personas para una sociedad competitiva, añadiendo incluso que el trabajo en grupo solo beneficia a los estudiantes con menor rendimiento académico (Velázquez, 2010).

6. Falta de control de aula:

Pujolás (2001) asegura que resulta complicado dar respuesta a "la presencia de algún alumno conflictivo en un equipo cooperativo, con poca motivación por el estudio, o con más ganas de importunar que de colaborar" (citado por Marín y Blázquez, 2003. p. 100). Además, añade que es mucho el personal docente que justifica la no utilización del trabajo en grupo por la dificultad de mantener el control en este tipo de clases.

7. Actitud o expectativas del cuerpo docente:

En ocasiones el propio docente pretende ver resultados casi inmediatos (Poveda, 2006). Como señala Rué (1998, citado por Marín y Blázquez, 2003), a veces el profesorado muestra una actitud ingenua acerca del trabajo cooperativo, del que solo espera beneficios, sin tener en cuenta los posibles problemas que puede encontrarse, y sin valorar la necesidad de tiempo y paciencia para dominar el uso de cualquier nueva metodología. Este desencanto, provocado por el fracaso de las primeras experiencias puede ser minimizado a través de un entrenamiento previo de los estudiantes en habilidades sociales o dinámica de grupos (León, 2002).

\section{Propuestas para la implementación del aprendizaje cooperativo}

Las investigaciones desarrolladas sobre el AC en EF han estado centradas en comparar la efectividad de esta metodología con otras, y en evaluar las mejoras o aprendizajes que a nivel motor, social o afectivomotivacional se consiguen con su aplicación. Son escasas las investigaciones que se centran en los problemas que se plantean, las posibles soluciones a dichos problemas, o las condiciones necesarias para maximizar los logros del AC (Velázquez, Fraile y López, 2014).

El libro publicado por Grineski (1996) es considerado como el primer manual que hace referencia de una manera explícita a la aplicación del AC en EF (Velázquez, 2013a). En él, además de proponer diferentes ejemplos de actividades y técnicas, se plantea un proceso de transformación pedagógica para ir desde enfoques más tradicionales hacia un enfoque cooperativo. Sin embargo, dado el carácter general del proceso expuesto por Grineski (1996), aún no podía considerarse como "una guía precisa que los docentes pudieran seguir" (Iglesias et ál., 2017, p. 274). 
Para este proceso de transformación, Grineski (1996) plantea una primea fase de modificación de las sesiones que el profesorado suele poner en práctica en su rutina habitual. Para ello, el o la docente ha de determinar los objetivos motores, cognitivos y afectivos que se plantea conseguir con las actividades grupales que ha diseñado. Mientras estas se llevan a cabo, observa a sus estudiantes trabajando, y comprueba si ejecutan la tarea de una manera individual o grupal, y si las oportunidades de participación y aprendizaje dependen de factores como la habilidad, género o suerte. Si la manera de estructurar la actividad planteada provoca que solo unos cuantos estudiantes consigan los objetivos previstos, y por lo tanto, que solo esos disfruten con la práctica, el profesorado debe elegir una estructura cooperativa para repetir la misma actividad, con el fin de aumentar el número de estudiantes que aprenden y disfrutan con esta. Con la nueva estructura de actividad se repite la misma observación, y en el caso de no funcionar debe intentarlo con otra.

Una vez que el cuerpo docente vaya sintiéndose más cómodo y competente modificando sus actividades a través del uso de estructuras cooperativas, se pasa a una segunda fase denominada creación de sesiones cooperativas. Para ello, debe seleccionar aquellas estructuras que se consideran más adecuadas en función de los objetivos planteados. A medida que se vaya ganando en experiencia, confianza y competencia en cooperación, se podrán modificar, e incluso, crear estructuras para adaptarlas a las necesidades de aprendizaje del alumnado. En esta transformación la evaluación del proceso de enseñanza-aprendizaje tiene gran importancia y significatividad. A través sobre todo de la observación y la autorreflexión, el profesorado va tomando decisiones y ejecutando los cambios pertinentes en el proceso de enseñanza-aprendizaje.

Continuando con la revisión bibliográfica sobre los procesos de aplicación del aprendizaje cooperativo en el aula, es bastante frecuente encontrar referencias relacionadas con el número de integrantes que forman los grupos de trabajo y la duración de estos. Iglesias et ál. (2017) señala que ambos son aspectos a tener en cuenta si se pretende desarrollar la metodología de una manera progresiva. Incluso para León (2002), es uno de los factores previos que van a determinar su eficacia. En general, se recomienda iniciar con grupos más reducidos, para ir incrementando progresivamente el número de integrantes, comenzando incluso el trabajo en parejas (Dyson, 2010; Iglesias et ál., 2017; Jacobs, 2006, citado por Ruiz, 2017). El número de interacciones posibles aumenta en función del número de integrantes del grupo. Cuanto más numeroso sea, mayor habilidad social deberán mostrar para coordinarse, para asegurarse de que todos cumplen su tarea, para brindarles a todos la oportunidad de expresarse, y para mantener las buenas relaciones de trabajo (Johnson et ál., 1999). Dyson (2010) recomienda empezar trabajando en parejas.

El éxito o fracaso en las primeras experiencias de AC puede ser un factor determinante para querer continuar o abandonar el camino de la cooperación. Grupos amplios al principio pueden generar una menor responsabilidad individual por parte de sus integrantes y una mayor dificultad para el profesorado a la hora de detectar los posibles problemas (Johnson et ál., 1999), y por lo tanto más dificultades para actuar sobre los problemas iniciales.

En resumen, consideramos escasas las propuestas que nos puedan servir a los docentes como guía a la hora de embarcarnos en este mundo tan interesante, y a la vez complicado en sus inicios, como es el de la cooperación. Nos referimos a propuestas que concreten una manera progresiva y eficaz de llevar a cabo la metodología en el aula, que definan las variables a influir, o las condiciones necesarias a generar, con el objetivo de minimizar o solucionar estos problemas iniciales. A continuación, se presentan de manera resumida las dos aportaciones encontradas que cumplen estos requisitos expuestos:

1. Coopedagogía Motriz (Velázquez, 2014, 2015, 2018)

La Coopedagogía, o pedagogía de la cooperación, se define como "el enfoque educativo orientado a promover que el alumnado aprenda a cooperar y utilice las posibilidades que la cooperación le ofrece para alcanzar eficazmente diferentes aprendizajes curriculares" (Velázquez, 2014, p. 45). A este enfoque centrado en el área de EF se le denomina Coopedagogía Motriz. 
Para su desarrollo plantea un proceso de cinco pasos, que tienen como finalidad cambiar la lógica del alumnado, promoviendo un entorno muy distinto al de tipo individualista o competitivo al que acostumbran.

El paso 1, creando conflicto, pretende generar en el alumnado procesos de reflexión a través de la práctica que les haga cuestionarse sus ideas previas. El conflicto cognitivo se produce entre lo que los estudiantes consideran normal en base a sus experiencias previas (actuar de manera individual o competitiva), y lo que cada docente considera que debería ser normal (ayudarse entre compañeros). De esta manera, a través de las actividades de estructura compartida y los procesos de reflexión generados a partir de estas, se pretende que el estudiantado comprenda que trabajar individualmente o competitivamente permitirá a algunos (pocos o muchos), pero no a todos, conseguir los objetivos planteados. Asociado a esto, se pretende generar una sensibilización en el estudiante que le lleve a querer aprender a cooperar.

Con el paso 2, denominado entendiendo la lógica de la cooperación, se busca generar un ambiente social de tipo cooperativo en el aula. Docente y estudiante identifican aquellas situaciones contrarias las cuales pueden darse en clase y se alejan de la lógica cooperativa, y establecen de manera consensuada el conjunto de normas de funcionamiento de la clase, desde la premisa de que nadie puede sentirse mal en la clase y que todos debemos preocuparnos de los demás.

Paralelo a esto se pretende que el alumnado vaya adquiriendo las habilidades necesarias para regular los conflictos que aparecen cuando se trabaja con otros. En esta parte se aconseja utilizar las siguientes estrategias didácticas: reforzar las actitudes de cooperación, vincular conductas y sentimientos en las reflexiones al finalizar la sesión, y elaborar procesos de autoevaluación y coevaluación, registrando la información, a los que poder acudir para dialogar con las y los alumnos.

En la fase 3, aplicando la lógica de la cooperación, el juego cooperativo es el principal recurso a utilizar, buscando que los grupos vayan resolviendo los problemas planteados por el mismo. El nivel de reto establecido en el juego por el o la docente será determinante para su éxito. Se continúa planteando procesos de reflexión después de las tareas ejecutadas, con el objetivo de seguir reforzando aquellas conductas de tipo cooperativo manifestadas por el estudiantado, y corregir aquellas contrarias.

Será en el paso 4, cuando se utilice el AC como metodología. Esta fase, aprendiendo a través del aprendizaje cooperativo, a su vez se puede dividir en otras dos. Una primera donde se utilizan técnicas muy sencillas que aseguran una interacción de las y los alumnos con una participación equitativa: Marcador colectivo (Orlick, 1990), Tres Vidas (Velázquez, 2004, 2013b) o Descubrimiento compartido (Velázquez, 2012, 2014); y una segunda donde se utilizan técnicas menos estructuradas en las que los estudiantes tienen una mayor responsabilidad a la hora de tomar decisiones: Puzle (Aronson, Blaney, Stephan, Sikes y Snapp, 1978), Enseñanza recíproca (Mosston, 1978) o Equipos de Aprendizaje (Grineski, 1996). A medida que se avanza en esta fase, las y los alumnos progresan en autonomía para organizarse.

El último paso, aprendizaje autónomo, tiene como finalidad que el estudiantado se incluya en proyectos voluntarios donde apliquen lo aprendido en EF, generalmente fuera del horario escolar, en los que el personal docente ya no regula el proceso de trabajo grupal.

2. El ciclo del Aprendizaje Cooperativo (Fernández, 2003)

En este libro publicado en 2017, se proponen tres fases para implementar el AC. Su propuesta es una adaptación de Pujolás (2009) y Moruno, Sánchez y Zariquiey (2011). En ella establecen tres fases para la puesta en práctica del AC en el aula, si bien como ellos mismos señalan "no se puede presentar un modelo cerrado para la implementación del aprendizaje cooperativo, aunque sí se 
pueden sugerir modos de actuación que luego deberán someterse a los correspondientes procesos de adaptación al contexto y a las necesidades del alumnado" (Iglesias et ál., 2017, p. 59).

La primera fase tiene como objetivo la creación y cohesión de grupo teniendo como finalidad complementaria mostrar al alumnado las ventajas de la cooperación. Es una fase donde los agrupamientos son flexibles, informales y variables, incluso agrupándose por parejas. Para conseguir los objetivos pretendidos en este primer escalón de la implementación, plantean utilizar dinámicas, entendidas como "procesos de interacción (intercambio, discusión o debate entre personas durante un tiempo limitado dentro de un ambiente de armonía y respeto, que son planteados mediante situaciones ficticias y que pretenden conseguir objetivos concretos" (Iglesias et ál., 2017, p. 64). Estas dinámicas son de dos tipos: dinámicas de creación de grupo y dinámicas de sensibilización sobre la importancia del trabajo en equipo. Explican y desarrollan para su puesta en práctica veintiséis dinámicas de creación de grupo y dieciséis de sensibilización.

En la segunda fase, denominada El aprendizaje cooperativo como contenido a enseñar, se plantean situaciones en las que los estudiantes deban ayudarse mutuamente. Para ello, las actividades presentadas han de permitirles desarrollar las destrezas cooperativas necesarias en un ambiente donde se fomente la ayuda recíproca y el apoyo mutuo. En comparación a la fase anterior los agrupamientos son más estables y duraderos en el tiempo (parejas estables o equipos de base). Estas actividades se concretan en técnicas o estructuras cooperativas simples. Se denominan simples, pues no se necesitan tomar decisiones en grupo a la hora de llevarlas a cabo, y por lo tanto no requieren de un desarrollo avanzado de las habilidades interpersonales. Además, son estructuras que generan la necesidad de colaborar, son de corta duración y fáciles de aprender y aplicar. Identifican y desarrollan una relación de veinticinco técnicas o estructuras que se pueden llevar a cabo en el aula. Es importante también señalar que a pesar de haber superado la primera fase, entienden que se debe continuar con la organización de actividades relacionadas con la creación y cohesión del grupo, y que la frecuencia e intensidad de estas actividades estará determinada por las necesidades encontradas en los grupos.

Por último, la fase tres: El aprendizaje cooperativo como recurso para enseñar y aprender. Aquí el AC ya es considerado un recurso para enseñar y aprender los contenidos curriculares. Además de los equipos base, hacen referencia a equipos de expertos. Se sigue utilizando, y por lo tanto, afianzando técnicas simples, que incluso pueden llegar a combinarse. Se da un salto al incluir técnicas más complejas, definidas estas como "macroactividades que se desarrollan durante varias sesiones de clase” (Iglesias et ál., 2017, p. 87). Además de esta cuestión temporal, son estructuras que requieren un alto dominio o desarrollo de las destrezas cooperativas. Identifican y desarrollan seis estructuras complejas, argumentando su selección por su facilidad para poder ser aplicadas por las y los profesores.

\section{CONSIDERACIONES FINALES}

Estamos de acuerdo con Ovejero (1990) cuando señala que "la cooperación no puede conseguirse de la noche a la mañana, pero es suficientemente útil como para que merezca realmente la pena” (p. 164). Como ha podido comprobarse a través de la revisión desarrollada, iniciarse en la metodología de la cooperación conlleva una serie de dificultades en sus inicios. Siendo uno de los objetivos de nuestro estudio, el conocimiento por parte del personal docente de estos problemas iniciales, puede ayudarle en cierta medida a preverlos y actuar sobre ellos, mejorando así la eficacia en la aplicación de la metodología.

Algunas resistencias pueden provenir de elementos contextuales, como pueden ser la oposición familiar, los aspectos organizativos del centro, o la excesiva competitividad e individualismo en la sociedad actual. Este contexto también se reproduce en la escuela, siendo habitual encontrar en las aulas estudiantes con ausencia 
de las habilidades interpersonales necesarias para compartir su trabajo, aprendizaje y responsabilidad con otros compañeros. Esta falta de habilidades necesarias para la cooperación, puede desembocar en experiencias negativas cuando se aplican metodologías que requieran trabajo en grupo, pudiendo convertirse en una resistencia más.

En lo que respecta al personal docente, la falta o escasa formación en el uso del aprendizaje cooperativo es un factor importante. En ocasiones, a esto se le suma una falta de realismo en sus expectativas previas, esperando resultados positivos de manera casi inmediata, y sin prever las posibles dificultades iniciales. Además, aparecen nuevos problemas de control de aula, surgidos de las nuevas responsabilidades e interacciones entre estudiantes.

Además, son escasas las propuestas publicadas que puedan servir de guía al profesorado que se inician en la pedagogía de la cooperación. En este sentido, siendo este el segundo objetivo del estudio, solo se han encontrado dos que expongan de una manera concreta y práctica los pasos necesarios para su desarrollo, estableciendo los objetivos en cada una de las fases y las estrategias didácticas para conseguirlos: El enfoque de la Coopedagogía Motriz (Velázquez, 2014, 2015, 2018), y el Ciclo del Aprendizaje Cooperativo (Fernández, 2003).

Si comparamos y relacionamos ambas propuestas, y añadimos algunas de las ideas aparecidas durante el desarrollo del artículo, podemos establecer una serie de principios de procedimiento, que nos sirvan como guía inicial para construir una propuesta de aplicación propia:

- Existe una progresión en cuanto a nivel de dificultad en el ámbito de lo social (habilidades interpersonales requeridas, necesidad de tomar decisiones grupales, etc.). Ocurre lo mismo en cuanto al número de integrantes en los grupos y la duración de estos.

- Al principio, resulta necesario demostrar al alumnado las ventajas, o la necesidad de cooperar, con el objetivo de que sean ellos mismos los interesados en trabajar de esta manera.

- El clima social de la clase es un factor fundamental a tener en cuenta. Se aplican estrategias didácticas concretas para influir en el ambiente de la clase, y para crear una lógica democrática y cooperativa del aula.

Para concluir, futuras líneas de investigación sobre los aspectos tratados en el artículo podrían contribuir a la mejorar la eficacia en el uso del AC. En este sentido, una vez conocidas las principales dificultades que el personal docente puede encontrarse al aplicarlo, podría ser de gran utilidad comprobar cuáles son las condiciones idóneas para aplicarlo con éxito.

En esta línea, nuestra tesis doctoral en curso, trata de comprobar cómo los procesos de evaluación formativa pueden contribuir a ello, promoviendo actitudes prosociales entre el estudiantado y aumentando su responsabilidad individual hacia la tarea y los compañeros.

\section{REFERENCIAS}

Aronson, E., Blaney, N., Stephan, C., Sikes, J., y Snapp, M. (1978). The jigsaw classroom. [El puzzle], Beverly Hills, CA: SAGE.

Dyson, B. (2002). The implementation of cooperative learning in an elementary school physical education program. Journal of Teaching in Physical Education, 22(1), 69-85. https://doi.org/10.1123/jtpe.22.1.69

Dyson, B. (2010). Un modelo híbrido de instrucción en Educación Física: integrando los modelos del aprendizaje cooperativo y los juegos tácticos. En C. Velázquez Callado (Coord.), Aprendizaje Cooperativo en Educación Física. Fundamentos y aplicaciones prácticas (pp. 99-115). Barcelona: Inde.

Fernández, J. (2003). El aprendizaje cooperativo en el aula de educación fisica para la integración en el medio social: análisis comparativo con otros sistemas de enseñanza y aprendizaje. [CD-ROM]. Valladolid: La Peonza.

Grineski, S. (1996). Cooperative learning in Physical Education. Champaign, IL: Human Kinetics. 
Iglesias, J. C., González, L. F., y Fernández, J. (2017). Aprendizaje cooperativo. Teoria y práctica en las diferentes áreas y materias del currículum. Madrid: Pirámide.

Johnson, D., Johnson, R., y Holubec, E. (1999). El aprendizaje cooperativo en el aula. Buenos Aires: Paidós.

León, B. (2002). Elementos mediadores en la eficacia del aprendizaje cooperativo: entrenamiento en habilidades sociales y dinámicas de grupo (Tesis doctoral). Universidad de Extremadura, España. Recuperada de: http://dehesa.une x.es/xmlui/handle/10662/463

Marín, S., y Blázquez, F. (2003). Aprender cooperando. El aprendizaje cooperativo en el aula. Merida: Dirección general de ordenación, renovación y centros.

Martín, G. (2014). Utilización del aprendizaje cooperativo para la transformación de los aprendizajes del alumnado y la formación continua de las maestras de un centro rural agrupado (Tesis doctoral). Universidad de Valladolid, España. Recuperada de http://uvadoc.uva.es/handle/10324/7536

Moruno, P., Sánchez, M., y Zariquiey, F. (2001). La red de aprendizaje. Elementos, procedimientos y secuencia. En J. C. Torrego (Coord.). Alumnos con altas capacidades y aprendizaje cooperativo. Un modelo de respuesta educativa (pp. 267-314). Madrid: Fundación SM.

Mosston, M. (1978). Enseñanza de la educación física. Del comando al descubrimiento. Barcelona: Paidós.

Orlick, T. (1990). Libres para cooperar, libres para crear. Barcelona: Paidotribo

Ovejero, A. (1990). El aprendizaje cooperativo. Una alternativa eficaz a la enseñanza tradicional. Barcelona: PPU.

Poveda, P. (2006). Implicaciones del aprendizaje de tipo cooperativo en las relaciones interpersonales y en el rendimiento académico (Tesis doctoral). Universidad de Alicante, España. Recuperada de http://rua.ua.es/dspace/handle/1 $0045 / 4110$

Prieto, L. (2007). El aprendizaje cooperativo. Madrid: PPC.

Pujolás, P (2009). 9 ideas clave. El aprendizaje cooperativo. Barcelona: Graó.

Ruíz, J.V. (coord.). (2017). Aprendizaje cooperativo en Educación Física. De la teoría a la práctica en situaciones motrices. Madrid: CCS.

Velázquez, C. (2004). Las actividades físicas cooperativas. Una propuesta para la formación de valores a través de la educación física en las escuelas de educación básica. México, D.F.: Secretaría de Educación Pública.

Velázquez, C. (2009). Los peligros en el proceso de introducción de actividades y metodologías cooperativas en educación física. La peonza. Revista de educación fisica para la paz, 4, 22-29. Recuperado de https://dialnet.uni rioja.es/servlet/articulo?codigo $=3233931$

Velázquez, C. (2010). Una aproximación al aprendizaje cooperativo en Educación Física. En C. Velázquez Callado (Coord.), Aprendizaje cooperativo en Educación Física. Fundamentos y aplicaciones prácticas. pp. 17-89. Barcelona: INDE.

Velázquez, C. (2012). La pedagogía de la cooperación en Educación Física. Laguna de Duero: Colectivo la Peonza.

Velázquez, C. (2013a). Análisis de la implementación del aprendizaje cooperativo durante la educación obligatoria en el área de Educación Física (Tesis doctoral). Universidad de Valladolid, España. Recuperado de http://uvadoc.uv a.es/handle/10324/2823

Velázquez, C. (2013b). La pedagogía de la cooperación en Educación Física. Armenia: Kinesis.

Velázquez, C. (2014). Coopedagogía. El enfoque de la pedagogía de la cooperación en Educación Física. En C. Velázquez, J. Roanes y F. Vaquero (Coords.). Actas del IX Congreso Internacional de Actividades Físicas Cooperativas. Vélez Málaga - Torre del Mar, 30 de junio a 3 de julio (44-60). Laguna de Duero (Valladolid): La Peonza.

Velázquez, C. (2015). Coopedagogía. El enfoque de la pedagogía de la cooperación en Educación Física. La peonza. Revista de Educación Física para la paz (nueva época), 10, 3-22. Recuperado de: https://dialnet.unirioja.es/serv let/articulo?codigo $=5367746$

Velázquez, C. (2018). El enfoque de coopedagogía como pieza clave en la transformación social. En E. LlorenteCatalán, y D. Martos-García (Eds.), Educación Fisica y pedagogía critica: propuestas para la transformación 
Raúl Martínez Benito, et al. El Aprendizaje Cooperativo en la clase de Educación Física: dificulta...

personal y social (pp. 273-293). Lleida-Valencia: Edicions de la Universitat de Lleida. Publicacions de la Universitat de Valéncia.

Velázquez, C., Fraile Aranda, A., y López, V.M. (2014). Aprendizaje cooperativo en Educación Física. Movimiento, 20(1), 239-259.

VerboProducciones. (2017). Viriato. Recuperado de http://www.verboproducciones.es/images/pdf/dossier_viriato. pdf

CC BY-NC-ND 